\title{
Optimizations of stent and tissue leaflets in a new surgical bovine pericardial valve
}

\author{
Jinmiao Chen ${ }^{1,2,3}$, Jun Yang ${ }^{4}$, Li Zhang ${ }^{4}$, Wenrui Ma ${ }^{1,2,3}$, Shuyang Lu ${ }^{1,2,3}$, Chunsheng Wang ${ }^{1,2,3}$, Tao Hong ${ }^{1,2,3}$ \\ ${ }^{1}$ Department of Cardiac Surgery, Zhongshan Hospital, Fudan University, Shanghai 200032, China; ${ }^{2}$ Shanghai Engineering Research Center of Heart \\ Valve, Shanghai 200032, China; ${ }^{3}$ Shanghai Institute of Cardiovascular Disease, Shanghai 200032, China; ${ }^{4}$ Shanghai Cingular Biotech Corporation, \\ Shanghai 201318, China \\ Correspondence to: Tao Hong; Chunsheng Wang. Department of Cardiac Surgery, Zhongshan Hospital, Fudan University, No. 180 Fenglin Road, \\ Xuhui District, Shanghai 200032, China. Email: hong.tao@zs-hospital.sh.cn; wang.chunsheng@zs-hospital.sh.cn.
}

Submitted May 28, 2019. Accepted for publication Sep 27, 2019.

doi: $10.21037 /$ jtd.2019.10.32

View this article at: http://dx.doi.org/10.21037/jtd.2019.10.32

\section{Introduction}

Compared with mechanical valves, bovine pericardial valves have satisfactory hemodynamic performance, low risk of infective endocarditis, and low incidence of thromboembolism without long-term anticoagulation treatment (1). Even after decades of continues improvements in tissue leaflets and bioprosthetic valve structure, the existing bioprosthetic valves still have some drawbacks. Although mid- and long-term clinical outcomes of bovine pericardial valves are satisfactory in the literature, the durability is still a major concern because of structural valve deterioration (SVD) (2-6). Another drawback is the malposition between the alloy wire and the stent, which is often due to the deformation of the stent structure after implantation. This may eventually lead to reduced effective orifice area (EOA) and patient-prosthesis mismatch.

To address the above-mentioned issues, we adopted certain new optimizations on the stent structure and the tissue leaflet matching to improve the valve stability and durability, reduce the leaflet dynamic stress during valve opening and closing, decrease the possibility and the degrees of triangular leaflet opening and increase the EOA. Here, we presented the details of optimizations of stent and tissue leaflets in a new surgical bovine pericardial valve and the presentative results of the in vitro studies.

\section{Methods}

\section{The design of a three-layer stent structure}

The stent of this new surgical bovine pericardial valve was composed as a three-layer structure (Figure 1A,B). The inner stent layer was made of polyester (PET). The middle stent layer was made of hard Elgiloy superalloy and the outer stent layer was also made of PET. The inner diameter of the outer stent layer was slightly smaller than the outer diameter of the middle stent layer before assembly. The end edges of both the outer and inner stent layers extended beyond the end edge of the middle stent layer, thus forming a groove structure from the end edges of the three stent layers. The inner and outer stent layers were manufactured by ultrasonic welding and the middle stent layer was manufactured by electronic welding. When hooping each ring, the welding points were separated from the each other.

\section{Improvement of the leaflet shape and matching}

The shape of the tissue leaflet was designed based on repetitious high-speed photography and determined by computer modeling and calculation. Based on the results of deflection and thickness tests, three bovine pericardial leaflets comparable in thickness and flexibility were selected and matched together in a valve.

\section{Valve assembly and redesign of the sewing ring}

The new surgical bovine pericardial valve consisted of a three-dimensional alloy wire, three bovine pericardial leaflets, a stent and a sewing ring. The three-dimensional alloy wire was shaped by a pre-set machine and then wrapped with a polyester cloth. For the stent assembly, the inner stent layer was first hooped with the middle stent 

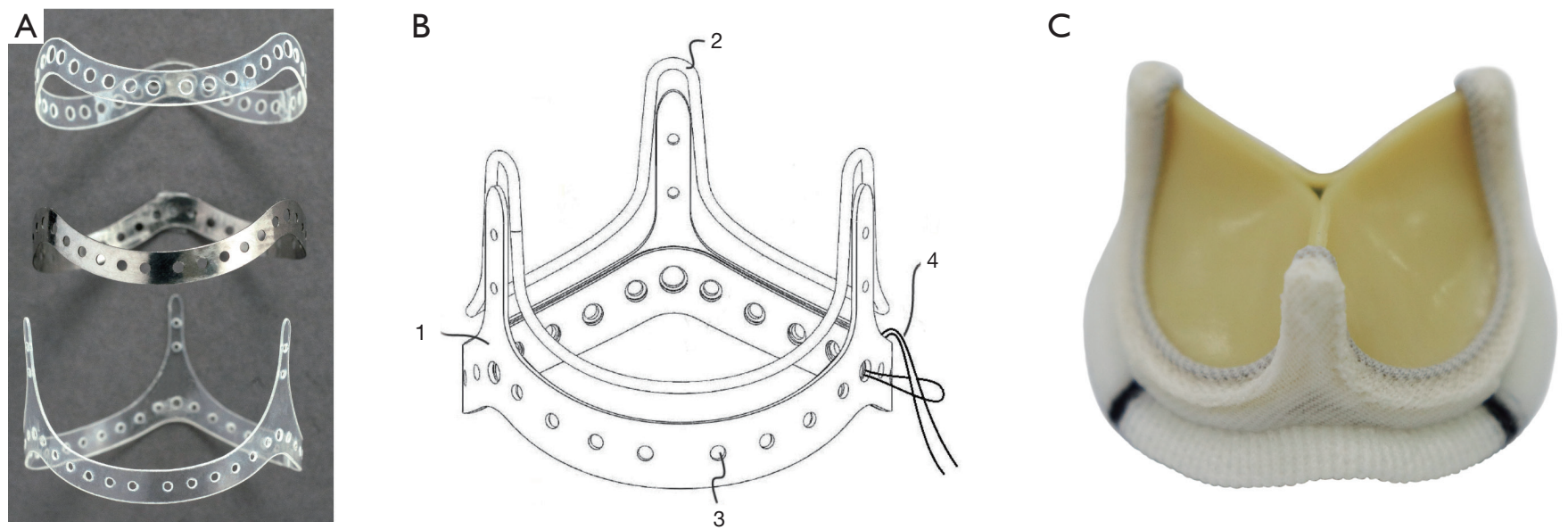

Figure 1 Structure of the new surgical bovine pericardial valve. (A) The three-layer stent structure of a new surgical bovine pericardial valve; (B) the three-dimensional structural view of the design of the new surgical bovine pericardial valve; (C) macro photograph of the finished new surgical bovine pericardial valve. 1 , stent; 2 , alloy wire; 3 , round holes; 4 , suture.

layer. Next the middle stent layer was hooped with the outer stent layer. Subsequently, the three layers of ring were fixed with suture through the round holes in pairwise alignment. The three-layer stent was wrapped with a polyester cloth. Finally, the composite structure including three bovine pericardial leaflets and the three-dimensional alloy wire were fixed to the groove formed by the three-layer stent. A redesigned sewing ring using Reemay with double velour Dacron was used in this new valve.

\section{In vitro evaluation of valve performance}

In vitro assessments were performed, including hydrodynamic performance and fatigue testing. For measurement of EOA, the following pulsatile-flow conditions were used: heart rate $=70$ cycles $/ \mathrm{min}$, simulated cardiac output $=5.0 \mathrm{~L} / \mathrm{min}$, and systolic duration $=35 \%$, at normotensive conditions. For fatigue testing, each test valve experienced a defined differential pressure $(100 \mathrm{mmHg}$ for aortic valve, $120 \mathrm{mmHg}$ for mitral valve) across the closed valve for at least $5 \%$ of each cycle during at least $95 \%$ of the test cycles. The frequency of this test was over 1,100 cycles/min.

\section{Results}

Assembly of the new surgical bovine pericardial valve was done by specially trained and skilled personnel. The presentative image of a finished Cingular valve was shown in Figure 1C. Due to the optimizations on the stent structure and the tissue leaflet matching, attachment and manufacturing, the improvement of the opening consistency and roundness made the EOA larger than the minimal requirements of the ISO 5840-2-2015. The EOA based on above pulsatile-flow conditions was evaluated in vitro (Figure 2 and Table 1). Fatigue testing demonstrated that this valve could remain functionally well for at least 200 million cycles.

\section{Discussion}

The current prosthetic heart valves are represented by two basic types. One is the mechanical valve, which is made of non-biologically originated materials. The other is the bioprosthetic valve, which is made of biological tissue material and mimicks the morphologic function and the working mechanism of the human heart valve itself. These two prosthetic heart valves have their own advantages and disadvantages. Compared with mechanical valves, bovine pericardial valves have satisfactory hemodynamic performance, low risk of infective endocarditis, and avoid long-term anticoagulation treatment (1). However, the bioprosthetic leaflets may become stiff or calcified over time after implantation. This is seen in particular at highstress areas of the leaflets, such as the junctions between the leaflets, the surrounding attachment points of the leaflets or the "cusps" at the outer edge of each leaflet. In addition, the leaflets are subject to continuous stress from valve opening and closing. So, the bioprosthetic valve will ultimately degenerate and require to be replaced by a new prosthetic valve. Since the prevalence of heart valve disease continues to increase as the global population ages and life expectancy is rising, an 

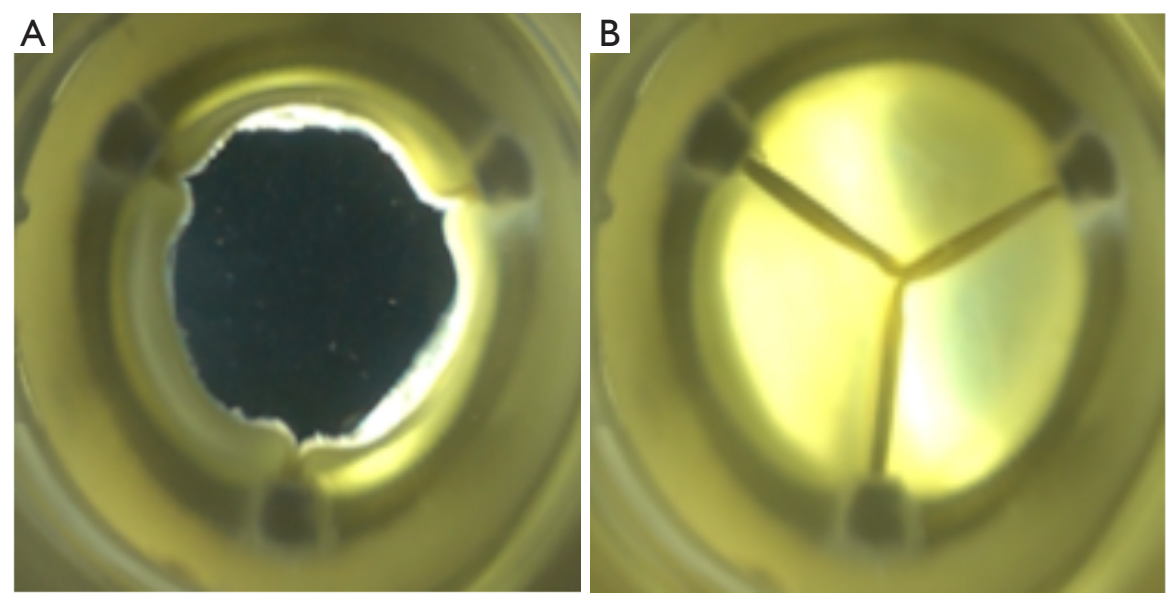

Figure 2 Macro photograph of valve opening during in vitro pulsatile-flow testing. Images of opening (A) and closing (B) of the valve.

Table 1 The EOA of different sizes of a new surgical bovine pericardial valve based on pulsatile-flow conditions

\begin{tabular}{lc}
\hline Size, $\mathrm{mm}$ & EOA (opening), $\mathrm{cm}^{2}$ \\
\hline 19 & $1.48 \pm 0.03$ \\
21 & $1.50 \pm 0.01$ \\
23 & $1.89 \pm 0.07$ \\
25 & $2.10 \pm 0.07$ \\
27 & $2.15 \pm 0.05$ \\
29 & $2.20 \pm 0.06$ \\
\hline
\end{tabular}

EOA, effective orifice area.

increasing need of bovine pericardial valves is foreseen (7). Thus, many commercially available prosthetic valves have undergone design modifications over the years (8-10).

The stent is fabricated from resilient material which is bent into a circular ring structure and whose ends are welded together. This manufacturing process makes the stent susceptible to a spring-back effect of the material and a tendency to undergo deformation. Because the stent is sewn together with the alloy wire, the stress on the alloy wire experienced during opening and closing of the valve makes the stent further susceptible to deformation, resulting in dislocation between the alloy wire and the stent. There is a gap between the alloy wire and the stent and the stent is oval rather than round. This malposition often leads to a shift of the alloy wire from the stent with a decreased stability of the entire valve, and uneven stress on the valve leaflets leading to wrinkles and expediting wear of the valve leaflets.

We designed a new surgical bovine pericardial valve to address the aforementioned shortcomings. The current valve adopted a three-layer stent structure. The advantage of this optimization represented improved stability of the valve in the following aspects: (I) The inner diameter of the external ring was slightly smaller than the outer diameter of the middle ring. The force produced by hooping with the external ring made the middle ring tend to be circular in both stationary and stressed states, so that good matching with the alloy wire is maintained. This innovation increased the overall stability of the valve and may extend its service life. (II) The inward or outward movement of the alloy wire can be restricted by the groove formed by the threelayer stent which made combination of the alloy wire and the stent tighter and the whole valve structure more stable. In addition, the leaflet shape and matching were further optimized, thus the possibility and the degrees of triangular leaflet opening were reduced. The preclinical trial in the juvenile ovine model showed that the new surgical bovine pericardial valve had similar mid-term satisfactory safety and efficacy compared with the Carpentier-Edwards Perimount ${ }^{\mathrm{TM}}$ valve in the mitral position of the juvenile sheep model (11). Long-term durability and performance are being accessed in the next studies.

\section{Acknowledgments}

We want to thank Peter Duijst for critically reviewing the manuscript.

Funding: This work is supported by the Shanghai Municipal Commission of Health and Family Planning (Grant No.201540385), Shanghai Sailing Program (Grant No.17YF1402100), Fudan University affiliated 
Zhongshan Hospital Excellent Youth Program (Grant No.2019ZSYQ05) and National Natural Science Foundation of China (Grant No.81801844).

\section{Footnote}

Conflicts of Interest: The authors have no conflicts of interest to declare.

Ethical Statement: The authors are accountable for all aspects of the work in ensuring that questions related to the accuracy or integrity of any part of the work are appropriately investigated and resolved.

\section{References}

1. Rahimtoola SH. Choice of prosthetic heart valve in adults an update. J Am Coll Cardiol 2010;55:2413-26.

2. Minakata K, Tanaka S, Okawa $Y$, et al. Long-term outcome of the carpentier-edwards pericardial valve in the aortic position in Japanese patients. Circ J 2014;78:882-9.

3. Bourguignon T, Bouquiaux-Stablo AL, Candolfi $\mathrm{P}$, et al. Very long-term outcomes of the Carpentier-Edwards Perimount valve in aortic position. Ann Thorac Surg 2015;99:831-7.

4. Johnston DR, Soltesz EG, Vakil N, et al. Long-term

Cite this article as: Chen J, Yang J, Zhang L, Ma W, Lu S, Wang C, Hong T. Optimizations of stent and tissue leaflets in a new surgical bovine pericardial valve. J Thorac Dis 2019;11(11):4855-4858. doi: 10.21037/jtd.2019.10.32 durability of bioprosthetic aortic valves: implications from 12,569 implants. Ann Thorac Surg 2015;99:1239-47.

5. Bourguignon T, Lhommet P, El Khoury R, et al. Very long-term outcomes of the Carpentier-Edwards Perimount aortic valve in patients aged $50-65$ years. Eur J Cardiothorac Surg 2016;49:1462-8.

6. Andreas M, Coti I, Rosenhek R, et al. Intermediate-term outcome of 500 consecutive rapid-deployment surgical aortic valve procedurest. Eur J Cardiothorac Surg 2019;55:527-33.

7. Iung B, Vahanian A. Epidemiology of valvular heart disease in the adult. Nat Rev Cardiol 2011;8:162-72.

8. Wyss TR, Bigler M, Stalder M, et al. Absence of prosthesis-patient mismatch with the new generation of Edwards stented aortic bioprosthesis. Interact Cardiovasc Thorac Surg 2010;10:884-7; discussion 887-8.

9. Wendt D, Stühle S, Piotrowski JA, et al. Comparison of flow dynamics of Perimount Magna and Magna Ease aortic valve prostheses. Biomed Tech (Berl) 2012;57:97-106.

10. Colli A, Marchetto G, Salizzoni S, et al. The TRIBECA study: (TRI)fecta (B)ioprosthesis (E)valuation versus (C)arpentier Magna-Ease in (A)ortic position. Eur J Cardiothorac Surg 2016;49:478-85.

11. Chen JM, Ding Y, Lu SY, et al. Noninferiority of Shanghai Cingular biotech's bovine pericardial valve preclinical study in juvenile ovine model. J Thorac Dis 2016;8:1179-87. 